Supporting information:

\title{
ZnO Nanoparticles Coated with Amphiphilic Polyurethane for Transparent Polyurethane Nanocomposites with Enhanced Mechanical and UV Shielding Performance
}

Shengwen Zhang*,†, Dandan Zhang ${ }^{\dagger}$, Huiyu Bai ${ }^{\dagger}$, Weihua Ming*,\$

The Key Laboratory of Food Colloids and Biotechnology, Ministry of Education, School of Chemical and Material Engineering, Jiangnan University, Wuxi 214122, China

Department of Chemistry and Biochemistry, Georgia Southern University, P.O. Box 8064, Statesboro, Georgia 30460, United States

*Corresponding author: zsw0825@yahoo.com,wming@georgiasouthern.edu. 
Table S1. Recipe of the synthesis of a series of PU with different end-capping agents.

\begin{tabular}{|l|c|c|c|c|c|c|}
\hline \multicolumn{1}{|c|}{ Sample } & \multicolumn{6}{|c|}{ Weight of each component (g) } \\
\hline \multirow{2}{*}{ PTMG-APU-BA } & PTMG & IPDI & DMPA & HEMA & BA & PETA \\
\cline { 2 - 7 } & 28.6 & 8.7 & 1.88 & 0 & 1.62 & 0 \\
\hline PTMG-APU-HEMA & 28.6 & 8.7 & 1.88 & 2.84 & 0 & 0 \\
\hline PTMG-APU-PETA & 28.6 & 8.7 & 1.88 & 0 & 0 & 6.51 \\
\hline
\end{tabular}




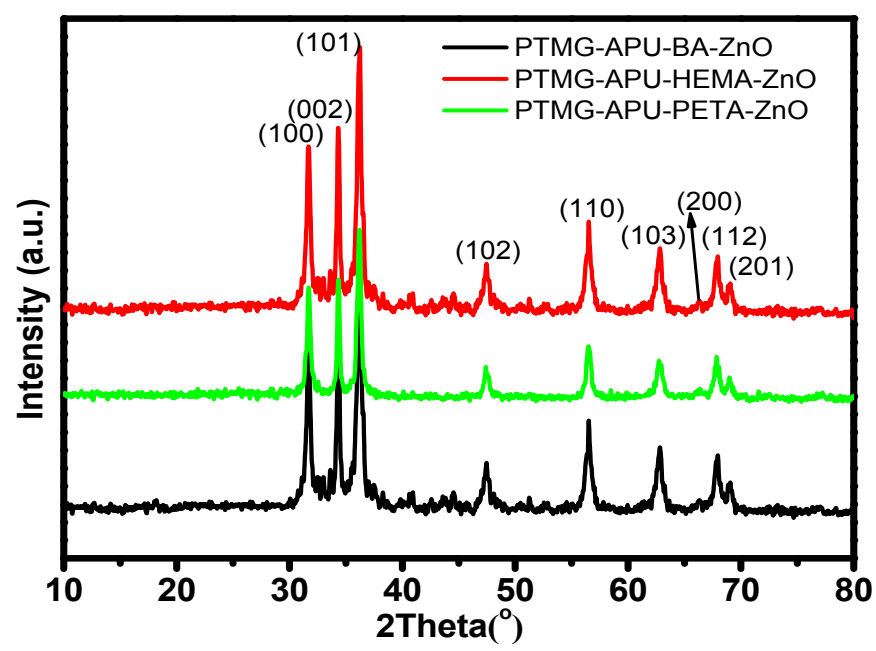

Figure S1. XRD spectra of APU-functionalized $\mathrm{ZnO}$ nanoparticles with different end-capping agents.

All the peaks, at $31.74^{\circ}, 34.38^{\circ}, 36.22^{\circ}, 47.54^{\circ}, 56.58^{\circ}, 62.90^{\circ}, 66.30^{\circ}, 67.92^{\circ}$, and $66.07^{\circ}$, are indexed to (100), (002), (101), (102), (110), (103), (200), (112), and (201) planes of hexagonal phase of $\mathrm{ZnO}$, respectively. The representative XRD patterns are consistent with the standard JCPDS card (NO.36-1451) of $\mathrm{ZnO}$ 